\title{
Der „unbestimmte Rechtsbegriff“ als Rechtsbegriff
}

- Zur normativen Kontrollfunktion der unbestimmten Rechtsbegriffe im technischwissenschaftlichen Zeitalter -

Auch wenn die dogmatische Notwendigkeit sowie die praktische Bedeutung des sog. „unbestimmten Rechtsbegriffs“ kaum noch umstritten sind, wird dieser im Zeitalter technischer Innovation und wissenschaftlicher Dynamik immer wieder als „Kronzeuge“ für die Steuerungsdefizite des Rechts bzw. des Gesetzes bezeichnet. ${ }^{1}$ Nach herkömmlicher Auffassung stellen die unbestimmten Rechtsbegriffe, die etwa im Bereich des Umwelt-, Technik- oder des Telekommunikationsrechts besonders häufig gebraucht werden, die Erkenntnisgrenzen des Gesetzgebers dar, so dass die substantialisierenden Konkretisierungsbefugnisse der jeweils zuständigen Behörde überwiegend in den Vordergrund gerückt werden müssten. ${ }^{2}$ In dieser Hinsicht versteht sich, weshalb und wie die Diskussionen um die unbestimmten Rechtsbegriffe immer wieder mit der Herausstellung der sachverständigen Standardsetzung oder der gestaltenden Ermessensentscheidung durch die Exekutive verbunden sind. Diese herrschende Auffassung zugunsten der „Gestaltungsaufgabe“ der Verwaltung hält die vorliegende Arbeit deshalb für unvertretbar, weil sie einseitig aus semantischen Gründen den unbestimmten Rechtsbegriff vorschnell rein als (rechtlich) inhaltsknappen und (außerrechtlich) konkretisierungsfähigen Begriff (miss)versteht und insofern dessen rechtlich bindende Bedeutung für die Verwaltung übersieht.

\section{Unbestimmter Rechtsbegriff als Schleusenbegriff?}

Im Gegensatz zur klassischen Vorstellung einer ,einzig richtigen Entscheidung“, welche allen unbestimmten Rechtsbegriffen immanent sei und letztlich von der umfassen-

1 So etwa Hoffmann-Riem, Ermöglichung von Flexibilität und Innovationsoffenheit im Verwaltungsrecht, in: ders./Schmidt-Aßmann (Hrsg.), Innovation und Flexibilität des Verwaltungshandelns, 1994, S. 9 ff., 27 ff.; Schmidt-Aßmann, Effizienz als Herausforderung an das Verwaltungsrecht - Perspektiven der verwaltungsrechtlichen Systembildung, in: Hoffmann-Riem/ ders. (Hrsg.), Effizienz als Herausforderung an das Verwaltungsrecht, 1998, S. 245 ff., 266 ff.; Rossen-Stadtfeld, Die verhandelnde Verwaltung - Bedingungen, Funktionen, Perspektiven, VerwArch 97 (2006), S. 23 ff., 32.

2 Vgl. dazu nur Trute, Die konstitutive Rolle der Rechtsanwendung, in: Trute/Gross/Röhl/Möllers (Hrsg.), Allgemeines Verwaltungsrecht - zur Tragfähigkeit eines Konzepts, 2008, S. 211 ff., 218 ff.; Fehling, Das Verhältnis von Recht und außerrechtlichen Maßstäben, in: Trute/Gross/Röhl/Möllers (Hrsg.), ebenda, S. 461 ff., 473 f. 
den gerichtlichen Überprüfung garantiert werde, ${ }^{3}$ geht heutzutage die herrschende Meinung davon aus, angesichts der technisch-wissenschaftlichen Entwicklungen und Fortschritten weise vor allem in den Referenzgebieten wie Umwelt-, Technik oder Telekommunikationsrecht der unbestimmte Rechtsbegriff kaum noch auf die lückenlose Verwaltungsgerichtsbarkeit, sondern vielmehr auf die erweiterte administrative Gestaltungsaufgabe hin. ${ }^{4}$ Dies spiegelt sich auf dogmatischer Ebene schon dadurch wider, dass gerade in diesen Referenzgebieten der behördliche Beurteilungsspielraum, innerhalb dessen die außerrechtlichen Erwägungen durch die Exekutive erlaubt und sogar gefordert sind, immer weiter anerkannt worden ist,${ }^{5}$ wobei die gerichtliche Überprüfung sich gewissermaßen auf die Kontrolle anhand rechtstaatlich allgemeingültiger Richtlinien beschränkt. ${ }^{6}$ Vor diesem Hintergrund versteht sich, warum besonders im technischwissenschaftlichen Zeitalter die unbestimmten Rechtsbegriffe gleichsam als „Schleusenbegriffe für metajuristische Standards"7 bezeichnet werden: Da immer mehr unbestimmte Rechtsbegriffe auf technisch-wissenschaftliche Sachkenntnisse sowie Bewertungen verweisen, welche offenbar über die rechtliche Auslegung und Anwendung hinausreichen, zeichnen sie sich immer häufiger durch ihre technisch-wissenschaftliche

3 Zur These der einzig richtigen Entscheidung vgl. schon Jesch, Unbestimmter Rechtsbegriff und Ermessen in rechtstheoretischer und verfassungsrechtlicher Sicht, AöR 82 (1957), S. 163 ff., 211 f., der davon ausging, bei der Ermessensentscheidung habe die Exekutive die Wahl zwischen mehreren Entscheidungen, von denen jede richtig sei, während es bei der Auslegung und Anwendung des unbestimmten Rechtsbegriffs nur eine richtige Entscheidung geben könne. Diese Annahme findet auch heutzutage noch und immer wieder Zustimmung. Vgl. z. B. Bleckmann, Spielraum der Gesetzesauslegung und Verfassungsrecht, JZ 1995, S. 685 ff., 686 f.; Maurer, Allgemeines Verwaltungsrecht, 17. Aufl., 2009, § 7, Rn. 29 f.; Peine, Allgemeines Verwaltungsrecht, 9. Aufl., 2008, § 4, Rn. 226 f. Obwohl diese klassische Voraussetzung einer „einzig richtigen Antwort“ für die Auslegung und Anwendung der unbestimmten Rechtsbegriffe seit langem als Fiktion entlarvt worden ist, wird bis heute allgemein angenommen, dass die unbestimmten Rechtsbegriffe materiell-inhaltlich grundsätzlich bestimmbar sein müssten, um das Verwaltungshandeln durch eine uneingeschränkte gerichtliche Überprüfung kontrollieren zu können und dadurch die rechtsstaatliche Ordnung sicherzustellen. Vgl. dazu näher Hwang, Gesetzesbindung durch unbestimmte Rechtsbegriffe: Eine kompetentielle Überlegung, VerwArch 102 (2011), S. 185 ff., $186 \mathrm{ff}$.

4 Vgl. nur den Überblick bei Hwang (Fn. 3), S. 185 ff., 194 ff.

5 Vgl. etwa Hoffmann-Riem, Reform des allgemeinen Verwaltungsrechts als Aufgabe - Ansätze am Beispiel des Umweltschutzes, AöR 115 (1990), S. 400 ff., 410 f.; Badura, Der atomrechtliche Funktionsvorbehalt der Genehmigungsbehörde für die Ermittlung und Bewertung des Risikos einer nuklearen Anlage, DVBl. 1998, S. 1197 ff., 1200 f.; Masing, Soll das Recht der Regulierungsverwaltung übergreifend geregelt werden?, Gutachten D zum 66. DJT, 2006, D 9 ff., S. 152 ff.; Trute, Das Telekommunikationsrecht - Eine Herausforderung für die Verwaltungsgerichte, in: Schmidt-Aßmann (Hrsg.), Festgabe 50 Jahre Bundesverwaltungsgericht, 2003, S. 857 ff., 859 f., $866 \mathrm{ff}$.

6 Vgl. dazu Schulze-Fielitz, Neue Kriterien für die verwaltungsgerichtliche Kontrolldichte bei der Anwendung unbestimmter Rechtsbegriffe, JZ 1993, S. 772 ff., 772 f.; Schoch, Gerichtliche Verwaltungskontrollen, in: Hoffmann-Riem/Schmidt-Aßmann/Voßkuhle (Hrsg.), Grundlagen des Verwaltungsrechts, Bd. , 2009, § 50, Rn. 296. Vgl. auch Jestaedt, Maßstäbe des Verwaltungshandelns, in: Erichsen \& Ehlers (Hrsg.), Allgemeines Verwaltungsrecht, 14. Aufl., 2010, S. 329 ff., 364 ff., 369 („Keinerlei Kontrollbesonderheiten bestehen im Blick auf die Auslegung des anzuwendenden Rechts.").

7 So Fehling (Fn. 2), S. 461 ff., 473 unter Berufung auf Schuppert, Verwaltungswissenschaft, 2000, S. 756. 
Konkretisierungsbedürftigkeit aus, wobei nicht (mehr) die Kontrollfunktion des Gerichts, sondern vielmehr die besondere Sachkunde der konkretisierenden Verwaltung im Mittelpunkt steht. ${ }^{8}$ Gerade aus dieser Perspektive heraus liegt es nahe, dass der unbestimmte Rechtsbegriff sich als Abgrenzungsbegriff darstellt, welcher rechtliche von außerrechtlichen Erwägungen unterscheidet: Indem die ersteren in den semantischen Bestimmungen des Gesetzgebers enthalten sind, müssten die letzteren erst und gerade der Verwaltung überlassen werden, die nicht nur im Vergleich mit dem Gesetzgeber, sondern auch gegenüber dem Gericht über eine „besondere fachliche Legitimation“ verfügt. Nach herkömmlicher Ansicht ist also festzustellen, dass der unbestimmte Rechtsbegriff sich nicht ausschließlich als „Rechtsbegriff“ darstellt, da er materiellinhaltlich immer wieder außerrechtlicher bzw. technisch-wissenschaftlicher Ergänzungen bedarf und schon mit diesem Charakter rechtlich nur wenig determinieren kann. ${ }^{9}$ Mit anderen Worten: Die materiell-inhaltliche Unbestimmtheit des unbestimmten Rechtsbegriffs sei erst durch die technisch-wissenschaftliche Vervollständigung zu kompensieren.

Das herkömmliche Verständnis des unbestimmten Rechtsbegriffs, welches sich auf die Gegenüberstellung von Recht und Nicht-Recht stützt, ${ }^{10}$ läuft indes Gefahr, angesichts der starken Abhängigkeit der dargelegten Referenzgebieten von technisch-wissenschaftlichen Kenntnissen zur Annahme zu führen, erst die sachverständige Exekutive sei in der Lage, den unbestimmten Rechtsbegriff zu substantialisieren und dergestalt dessen Steuerungskraft zu ,aktivieren“. Diese Ansicht würde den Eindruck erregen, der inhaltsknappe unbestimmte Rechtsbegriff könnte seine Steuerungsfähigkeit erst durch die behördliche substantielle Konkretisierung entfalten, die insofern an die Stelle des Gesetzes treten und daher auch die Reduzierung gerichtlicher Kontrolldichte herbeiführen müsste. Bereits die Kompetenzübertragung auf die Verwaltung beim unbe-

8 So z. B. Hoffmann-Riem, Eigenständigkeit der Verwaltung, in: Hoffmann-Riem/SchmidtAßmann/Voßkuhle (Hrsg.) (Fn. 6), Bd. , 2006, § 10, Rn. 90 ff., 126; Schulze-Fielitz, Einheitsbildung durch Gesetz oder Pluralisierung durch Vollzug, in: Trute/Gross/Röhl/Möllers (Hrsg.) (Fn. 2), S. 135 ff., 156 f. Vgl. auch Trute, Die konstitutive Rolle der Rechtsanwendung, in: Trute/Gross/Röhl/Möllers (Hrsg.), ebenda, S. 211 ff., 229 f., wo ein ,plurales Legitimationskonzept" zugunsten der Exekutive hervorgehoben wird.

9 Vgl. dazu etwa Oster, Normative Ermächtigungen im Regulierungsrecht. Eine vergleichende Untersuchung behördlicher Entscheidungsspielräume in der deutschen und amerikanischen Netzinfrastrukturregulierung, 2010, S. 54: „Bei den als ,unbestimmte Rechtsbegriffe“ bezeichneten Tatbestandsmerkmalen handelt es sich häufig nicht um Rechtsbegriffe wie Urteil, Diebstahl oder Ermessen, sondern um Begriffe aus anderen Wissenschaften, etwa der Psychologie, der Soziologie, der Naturwissenschaften, der Medizin oder der Wirtschaftswissenschaften.".

10 Bereits Carl Hermann Ule legte mit Nachdruck dar, die Verwaltungsbehörde sei „,in der Regel selbst sachverständig“, so dass der behördliche Beurteilungsspielraum schon aus funktionellrechtlicher Perspektive nicht auszuschließen sei: „Kann man in der Würdigung eines bestimmten Sachverhaltes zu verschiedenen Ergebnissen gelangen, was durch das Vorliegen verschiedener - gleichwertiger - Sachverständigengutachten nur unterstrichen wird, so hält sich jede dieser Würdigungen im Rahmen des unbestimmten Rechtsbegriffs und ist rechtmäßig. Dem Verwaltungsgericht sollte es deshalb verwehrt sein, in solchen Grenzfällen sein eigenes Werturteil an die Stelle des Urteils der Verwaltungsbehörde zu setzen.“ Ule, Zur Anwendung unbestimmter Rechtsbegriffe im Verwaltungsrecht, in: Bachof (Hrsg.), Gedächtnisschrift für Walter Jellinek, 1955, S. 309 ff., 326 (Hervorhebung im Original). 
stimmten Rechtsbegriff würde aber verfassungsrechtlichen Bedenken begegnen, denn sie verkennt den Bindungscharakter des unbestimmten Rechtsbegriffs, indem übersehen wird, dass der vom Gesetzgeber aufgestellte inhaltsoffene Steuerungsrahmen nicht durch die technisch-wissenschaftliche Substantialisierung ausgehöhlt werden darf. Anders ausgedrückt: Wird der unbestimmte Rechtsbegriff immer häufiger als Schleusenbegriff bezeichnet, so liegt die Gefahr nahe, dass der unbestimmte Rechtsbegriff immer weniger und schwieriger als Rechtsbegriff fungieren kann, der als gesetzliche Vorgabe und Maßstab das einzelne Verwaltungshandeln binden soll. Auch insofern ist die Ohnmacht des Gerichts nicht zu vermeiden, denn dieses ist ausschließlich zur rechtlichen Beurteilung berufen und muss daher immer bei außerrechtlichen Fragen auf seine Funktionsgrenzen stoßen. So gesehen steht fest, dass die Kategorisierung des unbestimmten Rechtsbegriffs in den Schleusenbegriff nicht nur die Gesetzesbindung erheblich schwächen, sondern darüber hinaus auch die gerichtliche Kontrollfunktion beträchtlich beeinträchtigen würde.

\section{Unbestimmter Rechtsbegriff als Rahmenbegriff}

Um die oben gewarnte Kompetenzverschiebung von der Legislative zur Exekutive zu verhindern, muss der unbestimmte Rechtsbegriff als Rahmenbegriff fungieren. Dies setzt voraus, dass die Gesetzesbindung sich nicht (immer) durch materiell-inhaltlich vollständige Vorbestimmung, sondern vornehmlich durch aufgabenbezogene und daher verpflichtende Ermächtigung vollzieht. ${ }^{11}$ Demgemäß lässt sich der unbestimmte Rechtsbegriff deshalb nicht unmittelbar für steuerungsunfähig halten, weil seine semantische Offenheit, die keineswegs bloß „Inhaltslosigkeit“ darstellt, sich auf die Verpflichtung der Exekutive zur Verwirklichung der ermächtigungskonformen Einzelfallgerechtigkeit richtet. ${ }^{12}$ Schon in dieser Hinsicht besteht die Aufgabe der gesetzesgebundenen Verwaltung nicht so sehr darin, in den einzelnen Entscheidungen die gesetzesimmanente substantielle Antwort herauszufinden, sondern vielmehr darin, den Zweck der jeweiligen gesetzlichen Ermächtigung zu erkennen und auf dieser Grundlage eine ermächtigungskonforme Entscheidung zu treffen. So betrachtet bedarf der unbestimmte Rechtsbegriff zwar weiterer Konkretisierung durch die Verwaltung, um im konkreten Einzelfall anwendbar zu werden. Die Anerkennung dieser behördlichen Konkretisierungsbefugnis ändert jedoch nichts daran, dass der konkretisierte unbestimmte Rechtsbegriff immer noch durch eine Rahmenordnung die zur Konkretisierung ermächtigte Verwaltung binden kann und soll.

$11 \mathrm{Zu}$ diesem Aspekt der Gesetzesbindung schon Kelsen, Reine Rechtslehre. Mit einem Anhang: Das Problem der Gerechtigkeit, 2. Aufl. (1960), Nachdruck 2000, S. 250. Zur Bindung gesetzlicher Ermächtigung vgl. auch Hwang, Rechtsbindung durch Rechtsermächtigung: Ein topisches Verständnis der reinen Rechtslehre zur Erläuterung des Verhältnisses von Richterbindung und Richterfreiheit, Rechtstheorie 40 (2009), S. 43 ff., 53 ff.; Spiecker Döhmann, Informationsgewinnung im Umweltrecht durch materielles Recht, DVB1. 2006, S. 278 ff., 279.

12 Vgl. insofern nur Merkl, Allgemeines Verwaltungsrecht, 1927, S. 152: „Der legislativpolitische Grund des Ermessens, soweit es gesetzlich bewusst eröffnet wird, ist nicht der, subjektiver Willkür Raum zu geben, sondern den besonderen Bedürfnissen des Einzelfalles Rechnung zu tragen.“. 
Das rahmenorientierte Verständnis der unbestimmten Rechtsbegriffe ist nicht lediglich dadurch zu rechtfertigen, dass besonders im technisch-wissenschaftlichen Zeitalter der Gesetzgeber nicht mehr in der Lage ist, die betreffenden Sachverhalte ex ante umfassend zu begreifen und vollständig zu regeln. Vielmehr spricht gerade die erhebliche Ungewissheit der zu regelnden Materie durchaus dafür, dass der Gesetzgeber der Verwaltung einerseits Entscheidungsspielräume zugunsten der Einzelfallgerechtigkeit einräumen, andererseits aber zugleich Entscheidungsregeln im Streben nach dem Zweck der jeweiligen Gesetzesordnung zur Verfügung stellen muss. Denn: Die technisch-wissenschaftliche Ungewissheit begründet nicht nur eine flexiblere und reaktionsfähigere Regelungsstrategie, sondern weist ferner darauf hin, dass - soweit das technisch-wissenschaftliche Wissen sich überwiegend als temporäres und daher unsicheres Wissen darstellt ${ }^{13}$ - auch die ,sachverständige ' Entscheidung der Verwaltung sich häufig nicht als objektiv „richtige“"Entscheidung erweisen kann. ${ }^{14}$ Bereits dies veranschaulicht, dass das Rechtssystem genau auf diese Ungewissheitsproblematik vornehmlich nicht mit immer weiterer Kompetenzübertragung auf die sachverständige Verwaltung, sondern vielmehr mit einer politischen Entscheidung zu reagieren hat. ${ }^{15}$ Diese muss in erster Linie feststellen, wie das vielfältige Wissen am Maßstab des Rechts zu behandeln ist bzw. worin die rechtlichen Grenzen der sachverständigen Verwaltungsentscheidung liegen. Mit anderen Worten: Vor allem unter Ungewissheitsbedingungen soll das Recht vornehmlich nicht dazu berufen sein, die technisch-wissenschaftlichen Maßstäbe selbst sachverständig und vollständig zu bestimmen, ${ }^{16}$ sondern vielmehr dazu, diesen Maßstäben normative Grenzen zu ziehen. Hinzu kommt, dass nicht die Exekutive, sondern die Legislative die begrenzende Rolle spielen soll, und zwar deshalb, weil nur sie im Rahmen der parlamentarischen Demokratie des Grundgesetzes in der Lage ist, die divergierenden technisch-wissenschaftlichen Gesichtspunkte oder Interessen im Wege einer demokratischen Willensbildung möglichst umfassend zu berücksichtigen und gerade dadurch die Freiheit möglichst vieler Menschen zu gewährleisten. ${ }^{17}$ Genau in dieser Hinsicht liegt es nahe, dass die semantische Unbestimmtheit der unbestimmten Rechtsbegriffe keineswegs auf deren Steuerungsunfähigkeit sowie Bindungslosigkeit, sondern

13 Vgl. Voßkuhle, Expertise und Verwaltung, in: Trute/Gross/Röhl/Möllers (Hrsg.) (Fn. 2), S. 637 ff., 652 ff.; Trute, Wissen - Einleitende Bemerkungen, in: H. C. Röhl (Hrsg.), Wissen - Zur kognitiven Dimension des Rechts, Die Verwaltung, Beiheft 9, 2010, S. 11 ff., 29; Röhl, Der rechtliche Kontext der Wissenserzeugung, in: ders. (Hrsg.), ebenda, S. 65 ff., 84.

14 Vgl. insofern auch Spiecker Döhmann (Fn. 11), S. 278 ff., 279.

15 Vgl. in diesem Zusammenhang auch Möllers, Kognitive Gewaltengliederung, in: Röhl (Hrsg.) (Fn. 13), S. 113 ff., 123: „Es geht nicht um einen definierten Wissensstandard, sondern darum, welche Lasten Grundrechtsträgern unter wie viel Ungewissheit zugemutet werden können.“.

16 Dabei wird freilich nicht die konkretisierende, maßstabsetzende Befugnis der Exekutive, sondern vielmehr deren Entgrenzung aufgrund einer angeblichen „sachlichen Legitimation“ verworfen.

17 Vgl. auch Zippelius, Politik und Sachverstand, in: Geis (Hrsg.), Planung - Steuerung - Kontrolle: Festschrift für Richard Bartlsperger zum 70. Geburtstag, 2006, S. 185 ff., 193 f.; Herdegen, Freistellung neuer Telekommunikationsmärkte von Regulierungseingriffen. Die gesetzliche Steuerung im Lichte des Verfassungs- und Europarechts, MMR 2006, S. 580 ff., 581; Hwang, Grundrechtsoptimierung durch (Kelsensche) Rahmenordnung: Zugleich ein Beitrag zur grundrechtsoptimierenden Funktion der unbestimmten Rechtsbegriffe am Beispiel „Stand von Wissenschaft und Technik“, Der Staat 49 (2010), S. 456 ff., 472 ff. 
vielmehr als verpflichtende Ermächtigung darauf hinweist, dass die Verwaltung ihre gesetzesbestimmte Aufgabe ermächtigungsgemäß erfüllen muss. Dabei dürfen niemals die selbst inhaltsknappen Regelungsgehalte, sondern vielmehr die rahmensetzenden Ermächtigungsgehalte der unbestimmten Rechtsbegriffe maßgeblich sein. ${ }^{18}$

Den vorstehenden Ausführungen ist zu entnehmen, dass auch und besonders im technisch-wissenschaftlichen Zeitalter der unbestimmte Rechtsbegriff immer noch als Rechtsbegriff dienen kann und soll. Wie dargelegt, begründet gerade die materiell-inhaltliche Offenheit des unbestimmten Rechtsbegriffs die notwendigen und doch vom demokratischen Gesetzgeber begrenzten Entscheidungsspielräume der Verwaltung. Diese ist zwar häufig nicht an die gesetzliche materiell-inhaltliche Vorentscheidung, wohl aber an die gesetzliche Rahmenordnung bzw. den Zweck sowie die Grenzen der Ermächtigung des jeweiligen unbestimmten Rechtsbegriffs zu binden. Dies verdeutlicht, dass nicht der materielle Inhalt, sondern der Ermächtigungsrahmen der Bindungskraft des unbestimmten Rechtsbegriffs zugrunde liegt, so dass dessen semantische Unbestimmtheit auch keine außerrechtliche Substantialisierung benötigt, um die Verwaltung binden zu können. Zugespitzt lässt sich sogar sagen, dass der unbestimmte Rechtsbegriff materiell-inhaltlich unbestimmt bleiben muss, um seine Bindungswirkung aufrechtzuerhalten. Denn: Gerade die von ihm bzw. seiner Unbestimmtheit geschaffene Rahmenordnung, die den Vorrang des rahmensetzenden Gesetzes erst verankert, ${ }^{19}$ bringt zum Ausdruck, welche normativen Grenzen die sachverständige Entscheidung der Verwaltung einzuhalten hat. So gesehen darf der Rechtscharakter des unbestimmten Rechtsbegriffs eben nicht durch eine technisch-wissenschaftliche Substantialisierung im Rahmen der Verwaltungsentscheidung ersetzt werden, denn dies würde nicht nur den Bindungsaspekt des ermächtigenden unbestimmten Rechtsbegriffs, sondern dadurch auch die Kompetenzabgrenzung zwischen Legislative und Exekutive beträchtlich relativieren. Auch in diesem Zusammenhang steht fest, dass erst das rahmenorientierte Verständnis des unbestimmten Rechtsbegriffs die Kontrollfunktion des Gerichts sicherstellt: Im Gegensatz zur herkömmlichen, substanzorientierten Vorstellung kennzeichnet der hier vertretene Ansatz den unbestimmten Rechtsbegriff als Rahmenbegriff, dessen Bindungskraft sich nicht durch materiell-inhaltliche Vorbestimmung, sondern durch verpflichtende Ermächtigung entfaltet. Demzufolge führt auch die Aussage, das Gericht sei ausschließlich zur Rechtskontrolle berufen, keineswegs zur Beschränkung gerichtlicher Überprüfung bei technisch-wissenschaftlichen Sachverhalten. Denn sowohl rechtliche als auch außerrechtliche Erwägungen müssen der Rahmenkontrolle des jeweilig ermächtigenden und zugleich verpflichtenden unbestimmten Rechtsbegriffs unterliegen. ${ }^{20}$ Am Beispiel des Umwelt- und des Telekommunikationsrechts wird im Folgenden gezeigt, weshalb und wie die semantisch offenen unbestimmten Rechtsbegriffe wie „Stand von Wissenschaft und Technik“ und „,wirksamer Wettbewerb“ als

18 Dazu näher Hwang (Fn. 3), S. 185 ff., 200 ff.

19 Zur Notwendigkeit einer Abstandwahrung zwischen Normen höherer und niederer Stufe vgl. ferner Hwang, Die Bindung des Richters an Gesetz und Recht: Richterliche Entscheidung nach dem richtigen Recht oder nach der Rahmenordnung des Rechts?, Rechtstheorie 38 (2007), S. $451 \mathrm{ff} ., 473 \mathrm{ff}$.

20 Vgl. dazu näher Hwang, Beurteilungsspielraum zugunsten außerrechtlicher Bewertung? Kritische Bemerkungen zur Einräumung behördlicher Beurteilungsspielräume im Umwelt- und im Telekommunikationsrecht, VerwArch (im Erscheinen). 
„Rechtsbegriffe“ fungieren sollen, um überhaupt ihre Bindungs- und Steuerungskraft zu entfalten.

\section{Stand von Wissenschaft und Technik als Rechtsbegriff}

Immer wieder wird der umweltrechtlich wohl bekannte Begriff „Stand von Wissenschaft und Technik“ als Einfallstor für außerrechtliche, insbesondere technisch-naturwissenschaftliche Erkenntnisse und Bewertungen bezeichnet. Die herkömmliche Lehre geht davon aus, dass die materiell-inhaltliche Unbestimmtheit des Begriffs im Grunde genommen auf eine Konkretisierungsbefugnis der Exekutive hinweise, die ,nicht nur gegenüber der Legislative, sondern auch im Verhältnis zu den Verwaltungsgerichten über rechtliche Handlungsformen [verfüge], die sie für die Verwirklichung des Grundsatzes bestmöglicher Gefahrenabwehr und Risikovorsorge sehr viel besser ausrüsten“. ${ }^{21} \mathrm{Zu}$ dem vertritt sie auch die funktionell-rechtliche Auffassung, die Verwaltung müsse bei der Konkretisierung dieses unbestimmten Rechtsbegriffs die technisch-(natur)wissenschaftlichen Fachkenntnisse einbeziehen, um ihre Entscheidung sachgerecht zu treffen. Bereits in dieser Hinsicht liegt es nahe, dass der unbestimmte Rechtsbegriff „Stand von Wissenschaft und Technik" sich deshalb auf den Beurteilungsspielraum bzw. die Einschätzungsprärogative der Verwaltung beziehe, weil erst diese durch hochspezialisierte Sachkompetenz in der Lage sei, den in Rede stehenden inhaltsknappen Begriff durch die Einbeziehung technisch-wissenschaftlichen Fachwissens zu substantialisieren und dergestalt seinen außer- bzw. nichtrechtlichen Charakter zu vergewissern ${ }^{22}$ - womit er seine Steuerungskraft erst richtig entfalte. Wie oben angedeutet wurde, lässt sich im Rahmen des Umwelt- und Technikrechts dieses große Vertrauen in den Sachverstand bzw. die sachverständige Verwaltungsentscheidung in erster Linie auf die allgemeine Angst vor erheblicher Ungewissheit zurückführen. Man hebt technisch-wissenschaftliches Fachwissen deshalb hervor, weil man Ungewissheit mit Wissen und besonders durch ständige Wissensgenerierung bekämpfen will. ${ }^{23}$ Daher betont etwa Liv Jaeckel, dass die Anerkennung autonomer Sachverhaltseinschätzungen auf „Sachlagen naturwissenschaftlich-technischer Ungewissheiten“ beschränkt werden solle. ${ }^{24}$ Diese Aussage versucht die Sachkompetenz der Verwaltung zwar einzuschränken, weist indes offenbar darauf hin, dass nicht das Gesetz, sondern erst und gerade die Verwaltung die

21 BVerwGE 72, 300 (317).

22 Exemplarisch Jaeckel, Gefahrenabwehrrecht und Risikodogmatik. Moderne Technologien im Spiegel des Verwaltungsrechts, 2010, S. $185 \mathrm{ff}$.

23 Zur Bekämpfung von Unsicherheiten und Ungewissheiten infolge der technisch-wissenschaftlichen Entwicklungen steht heutzutage der Versuch der Wissensgenerierung durchaus im Vordergrund. Vgl. z. B. Spiecker Döhmann (Fn. 11), S. 278 ff.; Vesting, Die Bedeutung von Information und Kommunikation für die verwaltungsrechtliche Systembildung, in: Hoffmann-Riem/Schmidt-Aßmann/Voßkuhle (Hrsg.) (Fn. 6), Bd. , 2008, § 20, Rn. 36 ff.; Röhl, Ausgewählte Verwaltungsverfahren, in: Hoffmann-Riem/Schmidt-Aßmann/Voßkuhle (Hrsg.), ebenda, § 30, Rn. 28 ff.; Fassbender, Wissen als Grundlage staatlichen Handels, in: Isensee/Kirchhof (Hrsg.), HStR, Bd. , 3. Aufl., 2006, § 76; Wollenschläger, Wissensgenerierung im Verfahren, 2009; Voßkuhle (Fn. 13), S. 637 ff., 656 ff., 663; Schuppert/Voßkuhle (Hrsg.), Governance von und durch Wissen, 2008; Röhl (Hrsg.) (Fn. 13).

24 Jaeckel (Fn. 22), S. 216. 
Verantwortung für die Ungewissheitsüberwindung tragen könne und solle. So gesehen liegt die Annahme nahe, der Rechtsbegriff „Stand von Wissenschaft und Technik“ müsse gewissermaßen selbst in den technisch-wissenschaftlichen Begriff umgesetzt werden, um dergestalt die Ungewissheit zu verringern. Eben insofern trete die Exekutive an die Stelle der Legislative auf der einen Seite und die Verwaltungsverantwortung an die Stelle der Verwaltungsgerichtsbarkeit auf der anderen Seite. ${ }^{25}$

Schon im Hinblick auf die Ungewissheitsproblematik selbst stellt sich jedoch die Frage, ob die erhebliche Ungewissheit infolge der starken Dynamik technisch-wissenschaftlicher Entwicklungen sich tatsächlich durch die stetige Wissensgenerierung auf untergesetzlicher Ebene mindern lässt. Zeichnet sich die Ungewissheitsproblematik genau dadurch aus, dass auch vorhandenes Wissen sich nicht selten als temporäres, unsicheres und sogar falsches Wissen erweist, ${ }^{26}$ so ist die Ungewissheit nicht mehr als Bekämpfungsgegenstand, sondern eher als gegebene Voraussetzung rechtlicher Regelungen anzusehen. ${ }^{27}$ Demzufolge entsteht allerdings der Zweifel, ob, weshalb und inwiefern die Verwaltung gerade unter den unüberwindbaren Ungewissheitsbedingungen noch über eine (im Vergleich mit dem Gesetzgeber stärkere) fachliche Legitimation verfügt, die zur Ungewissheitsbekämpfung immer wieder in den Vordergrund gerückt werden müsste. Zwar sind die Sachnähe sowie der Sachverstand der Verwaltung im Streben nach der Wissensgenerierung nicht zu leugnen. ${ }^{28}$ Doch steht unter Ungewissheit eben nicht das Wissen oder die Wissensgenerierung im Mittelpunkt. Denn selbst das Wissen müsste sich ständig auseinandersetzen, korrigieren und verändern, so dass auch die fachliche Legitimation der Verwaltung keine Aussagekraft für die ,exakte (Sach-)Richtigkeit“" entfalten kann. Vielmehr ist angesichts der Pluralität und Temporarität bzw. der Ungewissheit des Wissens das Recht vornehmlich dazu berufen, Rahmenbedingungen aufzubauen, die klar stellen sollen, welches Wissen für die rechtliche Regelung notwendig oder bedeutsam ist sowie auf welcher Grundlage und in welcher Weise das Wissen zu generieren ist. Aus dieser Perspektive ist daran festzuhalten, dass trotz und gerade wegen der Ungewissheitsproblematik die gesetzliche Bindung der Verwaltung unentbehrlich bleibt. Zwar setzt auf untergesetzlicher Ebene die Konkretisierung des Begriffs „Stand von Wissenschaft und Technik“ technisch-naturwissenschaftliche Erkenntnisse voraus, bei denen die Beurteilung sowie Einschätzung der zuständigen Behörde notwendigerweise eine bedeutende Rolle spielt. Dennoch ändert dies

25 In diese Richtung etwa Schulze-Fielitz (Fn. 6), S. 772 ff., 779; Hoffmann-Riem, Verwaltungsrechtsreform - Ansätze am Beispiel des Umweltrechts, in: Hoffmann-Riem/Schmidt-Aßmann/Schuppert (Hrsg.), Reform des allgemeinen Verwaltungsrechts: Grundfragen, 1993, S. 115 ff., 130 ff.; Kloepfer, Umweltschutzrecht, 2008, S. 46; Groß, Die Rezeption von Erkenntnissen der Wissenschaft und Technik im Verwaltungsrecht, in: Gropp/Lipp/Steiger (Hrsg.), Rechtswissenschaft im Wandel. Festschrift des Fachbereichs Rechtswissenschaft zum 400jährigen Gründungsjubiläum der Justus-Liebig-Universität Gießen, 2007, S. 153 ff., 156; Jaeckel (Fn. 22), S. 217 ff.

$26 \mathrm{Zu}$ diesem Aspekt der gegenwärtigen Ungewissheitsproblematik im Rahmen des Umweltrechts Jaeckel (Fn. 22), S. 16 ff. Vgl. auch Hwang, Normkonkretisierende Verwaltungsvorschriften im Umweltrecht: Normkonkretisierung als Normersetzung? KritV 2011, S. 97 ff., 111.

27 Insofern auch Vesting, Rechtswissenschaftliche Beobachtungen des Rechtssystems, in: Trute/ Gross/Röhl/Möllers (Hrsg.) (Fn. 2), S. 233 ff., 249.

28 Vgl. dazu etwa Möllers (Fn. 15), S. 113 ff., 125. 
nichts daran, dass bereits auf gesetzlicher Ebene dieser Begriff selbst einen Rahmen setzt, welcher zwar nicht durch substantiell festgelegte Standards, wohl aber durch verpflichtende Ermächtigungen die Verwaltungsentscheidung an bestimmte Aufgaben bindet. Daraus folgt, dass sowohl die technisch-wissenschaftlichen Sachkenntnisse als auch die daraus folgenden Bewertungen der Gesetzesbindung sowie der Gerichtskontrolle nach Maßgabe des Ermächtigungsrahmens dieses Begriffs unterliegen müssen.

So gesehen bindet der unbestimmte Rechtsbegriff ,Stand von Wissenschaft und Technik" die Verwaltung zuallererst dadurch, dass er keine inhaltsfesten technischwissenschaftlichen Standards, sondern lediglich einen Rahmen bestimmt, welcher gerade die genannte Unüberwindbarkeit der Ungewissheitsproblematik voraussetzt und infolgedessen die Verschiedenheit sowie Veränderbarkeit von Wissen gewährleisten will. Die Aussage, dass die Verwendung dieses Begriffs zum „dynamischen Grundrechtsschutz" ${ }^{29}$ dienen soll, soll daher nicht zu dem Missverständnis führen, dass die Behörde im Streben nach der (sich an einem dynamischen Grundrechtsschutz orientierenden) Einzelfallgerechtigkeit von der gesetzlichen Bindung losgelöst werden dürfe. Vielmehr stellt sie fest, dass die „Dynamisierung“ der Grundrechtsgewährleistung aus dem Gesetz bzw. dem Begriff, ,Stand von Wissenschaft und Technik“ erfolgt und daher vom Gesetzgeber dirigiert wird. Wenn also der Gesetzgeber den Begriff „Stand von Wissenschaft und Technik" verwendet, überlässt er der Behörde nicht einfach die sich aus deren eigenen Erwägungen ergebende endgültige Entscheidung. Er verpflichtet die Behörde vielmehr, sich nicht an einem bestimmten technischen oder wissenschaftlichen Gesichtspunkt, sondern vielmehr an einer pluralistischen Haltung zur Auseinandersetzung mit verschiedenen Wissenschaften zu orientieren. ${ }^{30}$ Genau in dieser Weise gewährleistet die begriffliche Verwendung von „Stand von Wissenschaft und Technik“ unter Voraussetzung der Unüberwindbarkeit der Ungewissheit die kognitive Freiheit möglichst vieler Interessen, ${ }^{31}$ da die Behörde nach dieser semantisch offenen Rechtsnorm dazu verpflichtet ist, alle ins Spiel kommenden technischen oder wissenschaftli-

29 BVerfGE 49, 89 (137).

30 Vgl. in diesem Zusammenhang auch Pitschas, Die Bewältigung der wissenschaftlichen und technischen Entwicklungen durch das Verwaltungsrecht, DÖV 1989, S. 785 ff., 792 f.; Plagemann/Tietsch, ,Stand der Wissenschaft“ und „Stand der Technik“ als unbestimmte Rechtsbegriffe, 1980, S. 29 ff., 36; Czajka, Der Stand von Wissenschaft und Technik als Gegenstand richterlicher Sachaufklärung, DÖV 1982, S. 99 ff., 108; Calliess, Rechtsstaat und Umweltstaat: zugleich ein Beitrag zur Grundrechtsdogmatik im Rahmen mehrpoliger Verfassungsrechtsverhältnisse, 2001, S. 216 ff., 245.

31 Nach Oliver Lepsius garantiert die kognitive Freiheit sogar „erst die Unsicherheit der Zukunft". Vgl. Lepsius, Prognose als Problem von Wissenschaft und Politik, in: Dreier/Willoweit (Hrsg.), Wissenschaft und Politik, 2010, S. 181 ff., 195 („Erst die Unsicherheit der Zukunft garantiert die kognitive Freiheit.“). 
chen Gesichtspunkte einzubeziehen und diese sachgemäß auszugleichen. ${ }^{32}$ Bereits in dieser Hinsicht verdeutlicht sich, dass der Begriff „Stand von Wissenschaft und Technik" weder Inhaltslosigkeit noch technisch-wissenschaftliche Fremdheit, sondern vielmehr einen rechtlich verbindlichen Gesetzesrahmen darstellt.

Dass der Begriff „Stand von Wissenschaft und Technik“ zur Gesetzesbindung der Verwaltung als Rechtsbegriff fungieren muss, weist ferner darauf hin, dass dieser Begriff im System unterschiedlicher gesetzlicher Regelungen unterschiedliche Ermächtigungsrahmen aufstellen könnte. Da die einzelnen Gesetze ihre eigenen Regelungsziele verfolgen, muss die Frage, zu welcher Aufgabenerfüllung die durch den Begriff „Stand von Wissenschaft und Technik" ermächtigte Verwaltungsentscheidung verpflichtet ist, anhand der demselben Begriff zugrunde liegenden Ermächtigungszwecke differenziert beurteilt werden. So betrachtet stellt sich der Rechtscharakter bzw. die gesetzliche Bindungskraft des Begriffs „Stand von Wissenschaft und Technik“ nicht lediglich durch dessen Gewährleistung kognitiver Freiheit dar, sondern darüber hinaus auch dadurch, dass dessen Ermächtigungsrahmen, der zur Bestimmung, Bindung und Kontrolle der Verwaltungsaufgabe beitragen soll, erst und gerade im Rahmen individueller gesetzlicher Regelungen differenziert zu ermitteln und festzustellen ist. Zum Beispiel müssen die Schutzgüter des Atom- und des Gentechnikgesetzes entsprechend dem Zweck des jeweiligen Gesetzes unterschiedlich bestimmt werden, ${ }^{33}$ so dass der unbestimmte Rechtsbegriff „Stand von Wissenschaft und Technik“ in den beiden Rechtsgebieten auf unterschiedliche Verwaltungsaufgaben verweisen muss: Während in $\S 7$ Abs. 2 Nr. 3 AtG die Anforderung nach dem „Stand von Wissenschaft und Technik“ angesichts der erheblichen Gesundheits-, Lebens- und Umweltschäden bei einem Reaktionsunfall deutlich ein besonders hohes Schutzniveau suggeriert, ${ }^{34}$ verpflichtet dieselbe Anforderung in $\S \S 11$ Abs. 1 Nr. 4 sowie 16 Abs. 1 Nr. 2 GenTG die Behörde dazu, vor allem unter Ungewissheit infolge stetig fortschreitenden Wandels von Wissenschaft und

32 Zur Aufgabe der Verwaltungsbehörde zur Interessenabwägung im Umwelt- und Technikrecht vgl. z. B. Di Fabio, Risikoentscheidungen im Rechtsstaat, 1994, S. 275, 286 ff.; Lange, Staatliche Steuerung durch offene Zielvorgabe im Lichte der Verfassung, VerwArch 82 (1991), S. 1 ff., 5 ff.; Groß (Fn. 25), S. 153 ff., 157; Appel, Methodik des Umgangs mit Ungewissheit, in: Schmidt-Aßmann/Hoffmann-Riem (Hrsg.), Methoden der Verwaltungsrechtswissenschaft, 2004, S. 327 ff., 336 ff. Gleichwohl steht im Rahmen dieser Diskussionen häufig nicht die Bindungskraft des parlamentarischen Gesetzes bzw. des unbestimmten Rechtsbegriffs, sondern vielmehr der (zu erweiternde) Beurteilungsspielraum der Verwaltung im Vordergrund.

33 Zum Unterschied zwischen Atom- und Gentechnikrecht vgl. Schmieder, Risikoentscheidungen im Gentechnikrecht. Beurteilungsspielräume der Verwaltung gegenüber den Gerichten?, 2004, S. 229 ff.; Beaucamp, Zum Beurteilungsspielraum im Gentechnikrecht, DÖV 2002, S. 24 ff., 26 ff.; Gärditz, Die Abwehr von Risiken gentechnischer Freisetzungen, ZUR 2009, S. 413 ff., 419.

34 So etwa Kloepfer (Fn. 25), S. 240, 243; Di Fabio (Fn. 32), S. 87; Marburger, Atomrechtliche Schadenvorsorge. Möglichkeiten und Grenzen einer normativen Konkretisierung, 2. Aufl., 1985, 19 f., 28 ff.; Sellner, Atom- und Strahlenschutzrecht. Ein abgeschlossenes oder zukunftsweisendes Kapitel höchstrichterlicher Rechtsprechung?, in: Schmidt-Aßmann (Hrsg.) (Fn. 5), 2003, S. 741 ff., 743, 746. 
Technik ${ }^{35}$ die verschiedenen und ggf. widerstreitenden technischen und wissenschaftlichen Meinungen miteinander abzuwägen, ${ }^{36}$ um dergestalt ein ,,verträgliches Nebeneinander zwischen dem Einsatz von neuartiger Gentechnik einerseits und den Schutzinteressen, dem Rechtsgüter- und Naturschutz andererseits ${ }^{\text {“37 }}$ zu gewährleisten. Gerade die unterschiedlichen Ermächtigungsgehalte, die sich aus den unterschiedlichen Ordnungsideen der jeweiligen Gesetze bzw. Gesetzesregelungen ergeben, spiegeln den Rechtscharakter des Begriffs „Stand von Wissenschaft und Technik“ deutlich wider.

Die Heranziehung des rechtlichen Aspekts und daher des (differenziert zu ermittelnden) Ermächtigungsgehaltes des Begriffs ,,Stand von Wissenschaft und Technik“ macht klar, dass erst dessen rechtlicher Rahmencharakter die Gesetzesbindung der Verwaltung garantiert. Die Rahmenordnung dieses Begriffs, die eben nicht durch die technischwissenschaftliche Substantialisierung auf untergesetzlicher Ebene verzerrt werden darf, setzt sich aber erst dann durch, wenn die gerichtliche Kontrollfunktion (wieder) ernst genommen wird. Wie dargelegt, spielt für die rechtliche Regelung unter Ungewissheit nicht das Wissen, sondern vielmehr die Gewährleistung seiner Vielfältigkeit und Veränderlichkeit die entscheidende Rolle. Schon an dieser Stelle verdeutlicht sich, dass die Verwaltungsgerichtsbarkeit nicht durch die (erhöhte) Verwaltungsverantwortung kompensiert oder gar ersetzt werden darf. Aus Sicht der gesetzlichen Rahmenordnung steht die Verwaltungsgerichtsbarkeit gerade dadurch im Vordergrund, dass der Begriff „Stand von Wissenschaft und Technik“ als Rechtsbegriff funktioniert: Unter technischwissenschaftlicher Ungewissheit stößt das Gericht deshalb nicht auf seine Funktionsgrenzen, weil nicht die - ihm fehlenden - technisch-wissenschaftlichen Fachkenntnisse, sondern vielmehr die Ermächtigungsrahmen dieses Begriffs als sein Kontrollmaßstab dienen, deren Ermittlung anhand der einzelnen Gesetzesregelungen seine Aufgabe sowie Kompetenz ist. In dieser Hinsicht liegt die Kategorisierung des Begriffs „Stand von Wissenschaft und Technik“ als Rechtsbegriff nicht nur der Gesetzesbindung, sondern auch der Gerichtskontrolle zugrunde.

\section{Wirksamer Wettbewerb als Rechtsbegriff}

Auch infolge seiner starken Abhängigkeit von technisch-wissenschaftlichen Entwicklungen zeichnet sich das Telekommunikationsrecht durch die häufige Verwendung von unbestimmten Rechtsbegriffen aus, die allgemeiner Meinung nach ohne interdisziplinäre Konkretisierungen nicht aufzufassen seien. Schon im Rahmen des Telekommunikationsgesetzes (TKG) tauchen zahlreiche unbestimmte Rechtsbegriffe wie ,(technische und wirtschaftliche) Tragfähigkeit“, „,effizient“, ,,verfügbare (Kapazität)“, „lang-

35 Vgl. z. B. Schmieder (Fn. 33), S. 46 ff., 190 f.; Röhl (Fn. 23), § 30, Rn. 29 f.; Hoffmann-Riem (Fn. 8), § 10, Rn. 128; Schulte/Apel, Recht der Umwelt- und Humangentechnik, in: Schulte/ Schröder (Hrsg.), Handbuch des Technikrechts, 2. Aufl., 2011, S. 505 ff., 509 f.; Gärditz (Fn. 33), S. 413 ff., 419.

$36 \mathrm{Zu}$ diesem Aspekt des Gentechnikrechts Schmieder (Fn. 33), S. 149 f.; Di Fabio (Fn. 32), S. 126 ff. („Nutzen-Risiko-Bilanz“ statt „klassische Gefahrdogmatik“).

37 Dies im Zusammenhang mit der Zwecksetzung des Gentechnikgesetzes Kloepfer (Fn. 25), S. 453 f.; Schmieder (Fn. 33), S. 86 ff.; Schubert, Zwanzig Jahre Gentechnikgesetz - eine Erfolgsgeschichte?, NVwZ 2010, S. 871 ff., $876 \mathrm{f}$. 
fristige (Sicherung des Wettbewerbs)“, „langfristige (Stabilität der wirtschaftlichen Rahmenbedingungen)“ und ,ausreichend“ immer wieder auf. Deren materiell-inhaltliche Unvollständigkeit wird deshalb weitgehend als Rechtsfertigungsgrundlage für die eigenständige Gestaltungsaufgabe der Verwaltung anerkannt, weil erst diese - genauso wie beim Umweltrecht - über eine besondere fachliche Legitimation ${ }^{38}$ verfügt. Auch das Bundesverwaltungsgericht betont: „Der Senat übersieht [...] nicht, dass die Konkretisierung unbestimmter Rechtsbegriffe grundsätzlich Sache der Gerichte ist, die die Rechtsanwendung der Verwaltungsbehörden uneingeschränkt $\mathrm{zu}$ überprüfen haben. Doch kann ein gesetzlich vorgegebenes Entscheidungsprogramm wegen hoher Komplexität oder besonderer Dynamik der geregelten Materie so vage und seine Konkretisierung im Nachvollzug der Verwaltungsentscheidung so schwierig sein, dass die gerichtliche Kontrolle an die Funktionsgrenzen der Rechtsprechung stößt. "39 Vor diesem Hintergrund überrascht es nicht, dass das den zahlreichen unbestimmten Rechtsbegriffen zu entnehmende „Regulierungsermessen“ 40 der Regulierungsbehörde, welches zur Rücknahme gerichtlicher Kontrolldichte führen solle, ${ }^{41}$ alles umfasst, was die Konkretisierung materiell-rechtlich vager und außerrechtlich komplexer unbestimmter Rechtsbegriffe betreffen soll. Auch und gerade der Zwecksetzung telekommunikationsrechtlicher Regulierung, einen wirksamen Wettbewerb herzu- und langfristig sicherzustellen, wohnt nach herkömmlicher Auffassung trotz der ausdrücklichen Definition des $\S 3$

38 So BVerwGE 130, 39 (48 f.); BVerwGE 131, 41 (62).

39 BVerwGE 130, 39 (48 f.). Vgl. in diesem Zusammenhang auch BVerfGE 84, 34 (50); BVerfGE 88, 40 (56); Danwitz, Die gerichtliche Kontrolle der Entgeltregulierung im Postund Telekommunikationsrecht, DVB1. 2003, S. 1405 ff., 1410. Dagegen Mayen, in: Scheurle/ ders. (Hrsg.), TKG Kommentar, 2008, § 21, Rn. 18.

40 Zum Begriff Masing (Fn. 5), S. 152 ff., 156; Ludwigs, Das Regulierungsermessen als Herausforderung für die Letztentscheidungsdogmatik im Verwaltungsrecht, JZ 2009, S. 290 ff., $291 \mathrm{ff}$.; Gärditz, „Regulierungsermessen“ und verwaltungsgerichtliche Kontrolle, NVwZ 2009, S. 1005 ff.; Schorkopf, Regulierung nach den Grundsätzen des Rechtsstaates. Zielvorstellungen guter Ordnung im neueren Verwaltungsrecht, JZ 2008, S. 20 ff., 24; Franzius, Wer hat das letzte Wort im Telekommunikationsrecht? Zum behördlichen Gestaltungsauftrag für die Zugangs- und Entgeltregulierung nach $\S \S 21,30$ TKG, DVBl. 2009, S. 409 ff., 410 ff.; Kersten, Herstellung von Wettbewerb als Verwaltungsaufgabe, in: VVDStRL 69 (2010), S. 288 ff., 322 ff.; Schneider, Rechtsschutz im Regulierungsverwaltungsrecht, in: Fehling/ Ruffert (Hrsg.), Regulierungsrecht, 2010, § 22, Rn. 19; Oster (Fn. 9), S. 158 ff.; Proelss, Das Regulierungsermessen - eine Ausprägung des behördlichen Letztentscheidungsrechts?, AöR 136 (2011), S. $402 \mathrm{ff} ., 411 \mathrm{ff}$.

41 So etwa BVerwGE 130, 39; BVerwGE 131, 41; BVerwG NVwZ 2009, 653. Zu dieser herrschenden Auffassung vgl. auch Schneider, Telekommunikation, in: Fehling/Ruffert (Hrsg.) (Fn. 40), § 8, Rn. 126 f.; Ziekow, Öffentliches Wirtschaftsrecht, 2. Aufl., 2010, § 14, Rn. 33. 
Nr. $31 \mathrm{TKG}^{42}$ ein interdisziplinärer Charakter inne, welcher zum behördlichen Regulierungsermessen führen müsste. „Ökonomische Rationalität ist hier die Leitlinie sowohl bei der Marktanalyse wie bei der vordergründig noch eher an die Struktur herkömmlicher Ermessensvorschriften erinnernden Instrumentenwahl. Angesichts des hiermit aufgegebenen gesetzlichen Entscheidungsprogramms bestehen kaum Zweifel, dass erhebliche Gestaltungsspielräume bestehen. Der Gesetzeswortlaut bringt dies punktuell mit drei durchaus traditionellen Figuren zum Ausdruck: erstens einem ,Beurteilungsspielraum' in $\S 10$ Abs. 2 TKG, zweitens einem Rechtsfolgen-, insbesondere Auswahlermessen z.B. in $\S 19,20,21$ und 24, und drittens einem zwischen Marktanalyse und Einschreiten abschichtenden, gestuften Entscheidungsprogramm. Da all diesen Elementen eine einheitliche Logik zu Grunde liegt: die situative Ausrichtung an ökonomischer Rationalität - dürfte das Kontrollkonzept hier weniger an den Einzelelementen (d.h. Beurteilungsspielraum und Rechtsfolgenermessen) auszurichten sein, als am übergreifenden Ganzen - d.h. einem spezifischen regulierungsrechtlichen Gestaltungsspielraum. “43 In dieser Hinsicht liegt es nahe, dass der Begriff des wirksamen Wettbewerbs in der Praxis weniger auf das gesetzliche Entscheidungsprogramm als auf die behördliche Entscheidungsprärogative verweist und sich insofern als interdisziplinären Begriff darstellt. 44

42 Nach $\S 3$ Nr. 31 TKG bedeutet wirksamer Wettbewerb die Abwesenheit von beträchtlicher Marktmacht im Sinne des $\S 11$ Abs. 1 Satz 3 bis 5 (TKG), wonach ein Unternehmen als Unternehmen mit beträchtlicher Marktmacht gilt, wenn es entweder allein oder gemeinsam mit anderen eine der Beherrschung gleichkommende Stellung einnimmt, das heißt eine wirtschaftlich starke Stellung, die es ihm gestattet, sich in beträchtlichem Umfang unabhängig von Wettbewerbern und Endnutzern zu verhalten. Die Bundesnetzagentur berücksichtigt dabei weitestgehend die von der Kommission aufgestellten Kriterien, niedergelegt in den Leitlinien der Kommission zur Marktanalyse und Ermittlung beträchtlicher Marktmacht nach Artikel 15 Abs. 2 der Richtlinie 2002/21/EG des Europäischen Parlaments und des Rates vom 7. März 2002 über einen gemeinsamen Rechtsrahmen für elektronische Kommunikationsnetze und -dienste (Rahmenrichtlinie) (ABl. EG Nr. L 108 S. 33) in der jeweils geltenden Fassung. Verfügt ein Unternehmen auf einem relevanten Markt über beträchtliche Marktmacht, so kann es auch auf einem benachbarten, nach $\S 10$ Abs. 2 bestimmten relevanten Markt als Unternehmen mit beträchtlicher Marktmacht angesehen werden, wenn die Verbindungen zwischen beiden Märkten es gestatten, diese von dem einen auf den anderen Markt zu übertragen und damit die gesamte Marktmacht des Unternehmens zu verstärken. Freilich weist die Vorschrift des $\S 11$ Abs. 1 TKG deutlich auf die Beurteilungs-, Einschätzungs- und Gestaltungsaufgabe der Regulierungsbehörde hin, deren Kompetenz also nicht nur zur Marktdefinition, sondern auch zur Marktanalyse eine schwerwiegende Rolle spielt. Vgl. insofern auch BVerwGE 131, 41 (44): „Die Abgrenzung des relevanten Marktes (§ 10 Abs. 1 TKG), die Prüfung seiner potenziellen Regulierungsbedürftigkeit ( 10 Abs. 2 Satz $1 \mathrm{TKG}$ ) sowie die Marktanalyse ( $\$ 11$ Abs. 1 TKG) sind aufeinander bezogen und untrennbar miteinander verbunden".

43 Spoerr, Der Einfluss ökonomischer Modellbildung auf rechtliche Maßstäbe der Regulierung, in: Trute/Gross/Röhl/Möllers (Hrsg.) (Fn. 2), S. 613 ff., 627.

44 Vgl. in diesem Zusammenhang auch Ludwigs (Fn. 40), S. 290 ff., 295: „Die hier befürwortete Kombination normtheoretischer und funktionell-rechtlicher Ansätze entbindet nun freilich nicht von der Anstrengung, konkrete Kriterien zu benennen, die im Einzelfall weiterführen. In den Fokus gerät dabei zunächst der interdisziplinäre, vielfach auf ökonomische Standards (wie die Effizienz) verweisende Charakter des Regulierungsrechts“. 
Es ist zwar nicht zu verleugnen, dass im Rahmen des TKG dem Begriff des wirksamen Wettbewerbs kein inhaltlich bestimmtes Leitbild zugrunde liegt, welches substantiell darauf hinweisen könnte, unter welchen Bedingungen und auf welche Weise das Ziel des wirksamen Wettbewerbs zu erreichen ist. ${ }^{45}$ Ebenso richtig scheint die Annahme, der Regulierungsbehörde stehe im Streben nach wirksamem Wettbewerb eine weitgehende Gestaltungsfunktion zu. Allerdings darf die gesetzliche Begriffsverwendung des wirksamen Wettbewerbs nicht schon deshalb als großzügige bzw. pauschale Kompetenzübertragung auf die Regulierungsbehörde missverstanden werden, deren umfangreiches Regulierungsermessen daher nicht gesetzliche Maßstäbe, ${ }^{46}$ sondern etwa „ökonomische Rationalität“ zu verfolgen hätte. ${ }^{47}$ Mit anderen Worten: Die Zwecksetzung eines „wirksamen Wettbewerbs“ lässt nicht alle Regulierungsmaßnahmen zu, die sich angeblich an Wettbewerbszielen orientieren. Nicht alle behördlichen Interventionen lassen sich im Namen des Wettbewerbs rechtfertigen. Vielmehr arbeitet der Gesetzgeber hier wiederum einen Ermächtigungsrahmen heraus, der gerade mit der Dynamik und Ungewissheit von Telekommunikationsmärkten rechnet und genau auf dieser Grundlage die Regulierungsbehörde dazu verpflichtet, ihre Regulierungsmaßnahme unter Berücksichtigung divergierender und ggf. widerstreitender Interessen und daher eher unter Notwendigkeitsbedingungen vorzunehmen. Dieser Bindungsaspekt des Wettbewerbsbegriffs spiegelt sich nicht nur dadurch wider, dass das Regulierungskonzept des TKG sich im Grunde genommen an einer ,regulierten Selbstregulierung“ orientiert, ${ }^{48}$ wobei

45 Vgl. etwa Pape, in: Geppert/Pipenbrock/Schütz/Schuster (Hrsg.), Beck'scher TKG-Kommentar, 2006, vor $\S 9$, Rn. 6: „Mit dem Begriff des wirksamen Wettbewerbs verfolgen der europäische und der deutsche Gesetzgeber kein wettbewerbspolitisches Leitbild, sondern umschreiben lediglich das Fehlen von beträchtlicher Marktmacht.“ Vgl. dazu auch Hwang, Wirksamer Wettbewerb durch offene Normen: Zum Funktionswandel der unbestimmten Rechtsbegriffe im Telekommunikationsrecht, AöR 136 (2011) (im Erscheinen).

46 Damit sei zwar nicht gemeint, dass das Regulierungsermessen gar keinen gesetzlichen bzw. rechtlichen Grenzen unterliegen müsste. Doch würden die gesetzlichen Rahmen im Streben nach dem wirksamen Wettbewerb materiell-inhaltlich so locker gesetzt, dass sie (aus Sicht der herkömmlichen substanzorientierten Ansätze) häufig nur noch die rechtsstaatlich allgemeingültigen Richtlinien wiedergeben könnten.

47 Vgl. z. B. Spoerr (Fn. 43), S. 613 ff., 636: „Wenn der Gesetzgeber in der Telekommunikationsregulierung die Übernahme eines bestimmten Wettbewerbsmodells abgelehnt hat, so hat er damit mehr und nicht weniger eine Ausrichtung an ökonomischer Rationalität und breitere Freiräume zugelassen“".

48 Vgl. schon Wegmann, Regulierte Marktöffnung in der Telekommunikation: Die Steuerungsinstrumente des Telekommunikationsgesetzes (TKG) im Lichte „regulierter Selbstregulierung“, 2001, S. 50 ff.; Hoffmann-Riem/Eifert, Regelungskonzepte des Telekommunikationsrechts und der Telekommunikationspolitik: Innovativ und Innovationsgeeignet?, in: Hoffmann-Riem (Hrsg.), Innovation und Telekommunikation. Rechtliche Steuerung von Innovationsprozessen in der Telekommunikation, 2000, S. 9 ff., 19. Vgl. auch Röger, Neue Regulierungsansätze im Telekommunikationsrecht - eine erste Analyse des neuen Telekommunikationsgesetzes 2004, DVBl. 2005, S. 143 ff., 152 f. Kritisch dazu Attendorn, Die Regulierungsbehörde als freier Marktgestalter und Normsetzer? Die Zugangsanordnung nach § 21 TKG im Vergleich zur Festlegungsentscheidung nach § 29 EnWG, 2008, S. 53 ff.; Attendorn, Regulierte Selbstregulierung: Gibt es das in der telekommunikationsrechtlichen $\mathrm{Zu}$ gangsregulierung?, DÖV 2008, S. 715 ff., 718 ff., der ablehnt, die telekommunikationsrechtliche (Zugangs-)Regulierung als Beispiel der „,regulierten Selbstregulierung“ aufzufassen. 
die Regulierungsbehörde zwar zur aktiven Marktbegleitung, ${ }^{49}$ keineswegs aber zur Marktersetzung berufen ist. ${ }^{50}$ Er spiegelt sich auch dadurch wider, dass - nach $\S \S 21$, 30 TKG - sowohl die Zugangs- als auch die Entgeltregulierung die Berücksichtigung vielgestaltiger Regulierungsziele und heterogener Interessen ${ }^{51}$ und insofern eine Verhältnismäßigkeitsprüfung voraussetzen müssen. ${ }^{52}$ Vor allem unter Ungewissheitsbedingungen spielt die Idee „regulierter Selbstregulierung“ eine wichtige Rolle, da sie besonders im Hinblick auf die hoch dynamischen Entwicklungen von Telekommunikationsmärkten gegen eine voraussetzungslos stark intervenierende Regulierungsstrategie spricht: Wenn die Technologien sich so schnell entwickeln, dass die Auswirkungen bestimmter Regulierungsstrategie nur schwer einzuschätzen sind, ${ }^{53}$ so kann die Regulierungsbehörde einen wirksamen Wettbewerb nur dann optimal fördern, wenn sie ihre Regulierungsmaßnahmen von technischem Wandel sowie heterogenen Wissensbeständen und Interessen abhängig macht und insofern der Selbstregulierung der Telekommunikationsmärkte genug Raum lässt. „Regulierung ist am wettbewerblichen Marktmechanismus ausgerichtet. Dieser soll optimiert, nicht aber durch hoheitliche Maßnahmen ersetzt werden. "54 Angesichts der Ungewissheitsproblematik gewährleistet der gesetzliche Ermächtigungsrahmen also keineswegs die Eigenständigkeit der behördlichen Regulierung, sondern in erster Linie die Selbstregulierung bzw. die Freiheit der Telekommunikationsmärkte. Auch in diesem Sinne ist die behördliche Regulierung durchaus gesetzesgebunden.

Aus dieser Perspektive verdeutlicht sich, dass der Begriff ,wirksamer Wettbewerb“ im Sinne des Telekommunikationsrechts sich nicht auf ein rein ermächtigendes, finales Gesetzesprogramm reduzieren lässt, bei dem schon der Wettbewerbszweck alle Mittel der Regulierungsbehörde begründen könnte. Die Rahmenordnung des wirksamen Wett-

49 Vgl. Hoffmann-Riem/Eifert (Fn. 48), S. 9 ff., 29: „Nach dem im TKG gewählten Ordnungsmodell übernimmt der Staat eine gewisse Verantwortung für die Marktgestaltung, insbesondere zur Beschränkung der Marktmacht des ehemaligen Monopolisten oder zukünftiger Marktbeherrscher, begrenzt sich aber im Wesentlichen auf eine aktive Marktbegleitung."Vgl. dazu ferner Hoffmann-Riem/Schulz/Held, Konvergenz und Regulierung, 2000, S. $50 \mathrm{ff}$.

50 Vgl. insofern auch Gärditz (Fn. 40), S. 1005 ff., 1008; Eifert, Regulierungsstrategien, in: Hoffmann-Riem/Schmidt-Aßmann/Voßkuhle (Hrsg.) (Fn. 6), Bd. , 2006, § 19, Rn. 138.

51 Im allgemeinen Kersten (Fn. 40), S. 288 ff., 327. Im Zusammenhang mit der Zugangsverpflichtungen Attendorn (Fn. 48/2008), S. 213 ff.; Piepenbrock/Attendorn, in: Geppert/Piepenbrock/Schütz/Schuster (Hrsg.) (Fn. 45), § 21, Rn. 75. Im Rahmen des Entgeltregulierung Mayen (Fn. 39), § 30, Rn. 28; insofern auch Scheurle, in: Scheurle/Mayen (Hrsg.) (Fn. 39), $\S 2, \mathrm{Rn} .1,6 \mathrm{ff}$.

52 Die Verhältnismäßigkeitsüberlegung spielt im Rahmen sowohl des $\S 21$ als auch des $\S 30$ TKG eine Zentralrolle. Vgl. etwa Jochum, Steht die Zugangsregulierung im Ermessen der Regulierungsbehörde für Telekommunikation und Post?, MMR 2005, S. 161 ff., 163; Attendorn (Fn. 48/2008), S. 203 ff. (zum § 21); VG Köln MMR 2007, S. 744 ff., 747; Mayen (Fn. 39), §30, Rn. 28, 51; ferner Groebel, Neuerungen im Bereich der Entgeltregulierung, K\&R Beiheft 1/2004, S. 18 ff., 18 f. (zu § 30).

53 Vgl. auch Ladeur, Die Regulierung von Selbstregulierung und die Herausbildung einer „Logik der Netzwerke": Rechtliche Steuerung und die beschleunigte Selbsttransformation der postmodernen Gesellschaft, in: Regulierte Selbstregulierung als Steuerungskonzept des Gewährleistungsstaates, Die Verwaltung, Beiheft 4, 2001, S. 59 ff., $62 \mathrm{f}$.

54 Bumke, Kapitalmarktregulierung. Eine Untersuchung über Konzeption und Dogmatik des Regulierungsverwaltungsrechts, Die Verwaltung 41 (2008), S. 227 ff., 229. 
bewerbs zeichnet sich vielmehr dadurch aus, dass sie von der oben beschriebenen Ungewissheit ausgeht und daher daran festhält, dass die konkreten Regulierungsmaßnahmen durch die Kooperation von Regulierung und Selbstregulierung ${ }^{55} \mathrm{zu}^{\mathrm{b}}$ bestimmen sind, wobei die Regulierungsbehörde zur umfassenden Abwägung von heterogenen Regulierungszielen und Interessen ${ }^{56}$ verpflichtet ist. Die Annahme, dass Wettbewerb im Regulierungsrecht „sowohl ein Regulierungsziel als auch ein Mittel zur Erfüllung der Gemeinwohlziele" ist, ${ }^{57}$ bekräftigt wiederum den Rechtscharakter des Wettbewerbsbegriffs: Dadurch, dass im Rahmen des Telekommunikationsgesetzes der wirksame Wettbewerb gemeinwohlorientiert ist,${ }^{58}$ wären behördliche Regulierungsmaßnahmen mit der Zwecksetzung wirksamen Wettbewerbs unvereinbar, wenn sie sich bereits unter Voraussetzung mangelnder gesetzlicher Steuerung vorschnell auf ökonomische Rationalität bzw. irgendwelche verabsolutierte Steuerungsansätze stützten. So gesehen sind nicht nur der wirksame Wettbewerb, sondern auch die sich auf den wirksamen Wettbewerb richtenden unbestimmten Rechtsbegriffe politische Wertentscheidungen des Gesetzgebers, ${ }^{59}$ die sich nicht kategorial vom Regulierungsermessen der Regulierungsbehörde abgrenzen, sondern dieses zur Erfüllung gesetzesbestimmter politischer Aufgaben verpflichten. Auch der Befund, dass sich die Entwicklung des Telekommunikationsrechts immer stärker am Gemeinschaftsrecht orientiert, ändert daran nichts, denn auch die Europäisierung telekommunikationsrechtlicher Regulierung zugunsten der eigenständigen Gestaltungsbefugnisse der nationalen Regulierungsbehörde (NRB) darf keineswegs die demokratischen und rechtsstaatlichen Vorbehalte des Gesetzes in Frage stellen. ${ }^{60}$ Daher kann die Aussage des Europäischen Gerichtshofs, dass ,auch die Abwägung zwischen [den gemeinschaftsrechtlichen Regulierungszielen] bei der Definition und der Analyse eines für die Regulierung in Betracht kommenden relevanten Marktes den NRB und nicht den nationalen Gesetzgebern [zusteht]“, ${ }^{61}$ nicht überzeugen.

55 Insofern auch Ladeur (Fn. 53), S. 59 ff., 74; Voßkuhle, „Regulierte Selbstregulierung“ - Zur Karriere eines Schlüsselbegriffs, in: ebenda, S. 197 ff., 199; Vesting, in: AK-GG, 3. Aufl., 2001, Art. 87 f., Rn. 29.

56 Zum Zielkonflikt innerhalb des Wettbewerbsziels vgl. ferner Lepsius, Ziele der Regulierung, in: Fehling/Ruffert (Hrsg.) (Fn. 40), § 19, Rn. 56 f.

57 So z. B. Lepsius (Fn. 56), § 19, Rn. 32.

58 Verfassungsrechtlich betrachtet ist die Gemeinwohlorientierung des Regulierungsrechts auf Art. 87 f. GG zurückzuführen, wobei der Schlüsselbegriff „Gewährleistungsstaat“ im Mittelpunkt steht. Vgl. dazu etwa Hoffmann-Riem, Telekommunikationsrecht als europäisiertes Verwaltungsrecht, DVB1. 1999, S. 125 ff., 126 f.; Masing, Post und Telekommunikation, in: Isensee/Kirchhof (Hrsg) (Fn. 23), § 90; Vesting (Fn. 55), Art. 87 f., Rn. 19 ff.

$59 \mathrm{Vgl}$. in diesem Zusammenhang auch Lepsius, Verfassungsrechtlicher Rahmen der Regulierung, in: Fehling/Ruffert (Hrsg.) (Fn. 40), § 4, Rn. 86.; Gärditz, Keine Regulierungsfreistellung für neue Märkte im Telekommunikationsrecht, JZ 2010, S. 198 ff., $199 \mathrm{ff}$.

60 So zutreffend Gärditz (Fn. 59), S. 198 ff., 200.

61 EuGH JZ 2010, 195 ff., 197, Rn. 91. Hierbei ging es um die Frage, ob die gesetzliche Regelung über die Regulierungsfreistellung nach $\S 9$ a TKG mit dem europäischen Telekommunikationsrecht vereinbar ist. Der EuGH hat die Vereinbarkeit verneint mit der Begründung, dass nicht der nationale Gesetzgeber, sondern die nationale Regulierungsbehörde über eine weitreichende Befugnis verfüge, ,um die Regulierungsbedürftigkeit eines Marktes in jedem Einzelfall beurteilen zu können“" (ebenda, Rn. 78). 
Dem Vorhergehenden ist zu entnehmen, dass die gerichtliche Kontrollaufgabe auch im Telekommunikationsrecht nicht durch die pauschale Anerkennung des sog. Regulierungsermessens ausgeblendet werden darf. Wie dargelegt, verweisen die zahlreichen unbestimmten Rechtsbegriffe nicht auf eine „Entpolitisierung“ der telekommunikationsrechtlichen Regulierung, sondern bringen im Gegenteil die politischen Entscheidungen des Gesetzgebers zum Ausdruck. Diese orientieren sich nicht nur am gemeinwohlbezogenen Wettbewerbsziel, sondern beschränken genau zu diesem Zweck die Regulierungsmittel der zuständigen Behörde. Demzufolge ist das Gericht durchaus zur Überprüfung berufen, ob die einzelnen behördlichen Regulierungsmaßnahmen den gesetzgeberischen politischen Wertentscheidungen entsprechen, wobei die aufgabenbezogenen Ermächtigungsrahmen der betreffenden unbestimmten Rechtsbegriffe maßgeblich sind. So gesehen spricht der Rechtscharakter des „wirksamen Wettbewerbs“ deutlich dagegen, die gerichtliche Kontrolldichte bei behördlichen Regulierungsermessen zu reduzieren.

\section{Fazit}

Die vorstehende Darstellung hat gezeigt, dass im technisch-wissenschaftlichen Zeitalter der interdisziplinäre Aspekt der unbestimmten Rechtsbegriffe vor allem bei der Rechtsanwendung wiederholt hervorgehoben wird. Zum Beispiel hat Hans-Heinrich Trute betont: „Methodisch weiß man über die normative Konstruktion des Wissens verhältnismäßig wenig. Jedenfalls aber kann man wissen, dass diese Konstruktionen kaum mit der Differenzierung von Rechts- und Tatsachenfragen behandelt werden können, auch nicht mit der Differenzierung von Rechtsfragen und sachverständiger Expertise. Diese Konstruktionen sind vielmehr ihrerseits in hohem Maße von rechtlichen ebenso wie wissenschaftlichen Konventionen geprägt [...]. “62 Diese Annahme veranschaulicht einen klaren Widerspruch: Während die herkömmliche interdisziplinäre Auffassung auf der einen Seite die Untrennbarkeit von rechtlichen und außerrechtlichen Fragen voraussetzt, um die behördliche Heranziehung von technisch-wissenschaftlichen Erkenntnissen zu begründen, hält sie auf der anderen Seite an der (der Lehre des Beurteilungsspielraums zugrunde liegenden) Gegenüberstellung von rechtlichen und außerrechtlichen Erwägungen fest, um die gerichtliche Überprüfung auf die Rechtskontrolle zu beschränken. Vielleicht könnte man dies mit der sog. funktionell-rechtlichen Überlegung erklären, wonach niemals das Gericht, sondern nur die Behörde zur außerrechtlichen Abwägung befugt sei. Gerade diese Kompetenzaussage zugunsten der Exekutive verursacht aber erhebliche Bedenken, da außerrechtliche Abwägungen im technischwissenschaftlichen Zeitalter einerseits zum Alltag geworden sind und sich andererseits von der Gerichtskontrolle abgrenzen lassen. Diese irreführenden Entwicklungen gehen darauf zurück, dass der unbestimmte Rechtsbegriff für inhaltslos gehalten wird. Daher ist es kein Wunder, dass man gelegentlich von „Bedeutungsverlusten juristischer Methodik" gesprochen hat. ${ }^{63}$ Wie aber in der vorliegenden Arbeit dargelegt wurde, stößt die herkömmliche Lehre auf Schwierigkeiten, da sie von Anfang an den Rechts- bzw.

62 Trute (Fn. 8), S. 211 ff., 227.

63 So Schulze-Fielitz (Fn. 8), S. 135 ff., 158. 
Rahmencharakter des unbestimmten Rechtsbegriffs und daher dessen materiell-inhaltliche Offenheit missdeutet. Wie gezeigt, kann der unbestimmte Rechtsbegriff deshalb als Rechtsbegriff fungieren, weil er seinem Wesen nach nicht durch substantielle Vorbestimmung, sondern durch Ermächtigung seine normative Bindungskraft entfaltet, welche eine lückenlose Gerichtskontrolle und einen effektiven Rechtsschutz ermöglicht. Besonders unter Ungewissheitsbedingungen darf der unbestimmte Rechtsbegriff nicht als etwas Sachfremdes beiseitegelassen werden, denn er geht gerade der gegebenen Ungewissheit der zu regelnden Sachverhalte voraus und stellt infolgedessen eine politische Entscheidung des Gesetzgebers dar, die die sachverständige Verwaltungsentscheidung nicht nur begründet, sondern auch begrenzt. Die rechtlichen und außerrechtlichen Erwägungen sind in diesem Sinne untrennbar: Sowohl erstere als auch letztere müssen an die ermächtigenden Gesetze, deren Ermächtigungsgehalt die Verwaltungsaufgaben bestimmt und zugleich begrenzt, gebunden werden. 